\title{
Quedas e qualidade de vida relacionada à saúde em idosos: influência do tipo, frequência e local de ocorrência das quedas
}

\author{
Falls and health-related quality of life: influence of frequency \\ and location type of falls
}

\begin{abstract}
Mariana Mapelli de Paiva (https://orcid.org/0000-0003-4947-7523) 1,2
Margareth Guimarães Lima (https://orcid.org/0000-0001-6996-0745) ${ }^{1}$

Marilisa Berti de Azevedo Barros (https://orcid.org/0000-0003-3974-195X) ${ }^{1}$
\end{abstract}

${ }^{1}$ Departamento de Saúde Coletiva, Faculdade de Ciências Médicas, Universidade Estadual de Campinas. R. Tessália Vieira de Camargo 126, Cidade Universitária Zeferino Vaz. 13083-887 Campinas SP Brasil.marianamapelli@ hotmail.com

${ }^{2}$ Instituto Federal do Norte de Minas Gerais, Campus Almenara. Almenara MG Brasil.

\begin{abstract}
This article aims to evaluate health-related quality of life (HRQOL) according to the frequency and characteristics of falls in the elderly. A cross-sectional study was carried out with data from a household health survey conducted in 2014 and 2015, in Campinas, SP. The dependent variables were the SF-36 domains and, the independent ones, the occurrence and the characteristics of the falls. The mean and mean differences of the SF-36 scores were estimated by simple and multiple linear regression. The study reveals that the impact on HRQOL depends significantly on the characteristics of falls. Larger declines and a greater number of SF-36 domains were observed in the elderly who suffered three or more falls (compared to those with one or two falls), reporting falls from fainting/dizziness (compared to falls due to tripping), those who fell at home (versus falls occurring elsewhere) and reported falling limitation in daily activities. Only in the falls with these characteristics did the domain of emotional aspects be affected. The impact on HRQoL, including emotional aspects, depends on the characteristics of the falls that need to be considered in prevention actions and in the control of the consequences of falls in the quality of life of the elderly.
\end{abstract}

Key words Quality of life, Accidents due to falls, Elderly
Resumo O objetivo deste artigo é avaliar a qualidade de vida relacionada à saúde (QVRS) segundo a frequência e as características das quedas em idosos. Trata-se de um estudo transversal desenvolvido com dados de inquérito domiciliar de saúde realizado em 2014 e 2015, em Campinas, São Paulo. As variáveis dependentes foram os dominios do SF-36 e, as independentes, a ocorrência e as características das quedas. As médias e as diferenças de médias dos escores do SF-36 foram estimadas por regressão linear simples e múltipla. O estudo revela que o impacto na QVRS depende significativamente das características das quedas. Declínios de maior magnitude e em maior número de dominios do SF-36 foram constatados nos idosos que sofreram três ou mais quedas (em comparação com aqueles com uma ou duas quedas), nos que relataram quedas por desmaio/ tontura (comparando com quedas provocadas por tropeção), nos que caíram no domicílio (versus as quedas ocorridas em outros locais) e nos que relataram limitação decorrente das quedas nas atividades diárias. Apenas nas quedas com essas características o domínio de aspectos emocionais se mostrou afetado. O impacto na QVRS, inclusive nos aspectos emocionais, depende das características das quedas, que precisam ser consideradas nas ações de prevenção e no controle das consequências das quedas na qualidade de vida dos idosos.

Palavras-chave Qualidade de vida, Acidentes por quedas, Idosos 


\section{Introdução}

O aumento da longevidade, que representa uma das maiores conquistas da humanidade, fez nascer um cenário em que afloram desafios para a preservação da independência e da qualidade de vida dos idosos nos anos de vida ganhos'.

Com o crescimento acelerado da população idosa, vários temas da área de geriatria e gerontologia ganharam proeminência, destacando-se entre eles as quedas e suas consequências ${ }^{2}$. A elevada frequência de quedas nos idosos resulta de alterações que se acentuam progressivamente com o avançar da idade, que incluem perda de massa, força e função muscular e perdas funcionais e nos sistemas sensorial, vestibular e nervoso. Essas perdas levam ao comprometimento da marcha e do equilíbrio, facilitando a ocorrência de quedas. Além do processo de envelhecimento, e também em decorrência dele, os idosos passam a apresentar uma incidência crescente de doenças crônicas que, junto com a consequente polifarmácia e seus efeitos adversos, podem aumentar o risco de quedas ${ }^{1,3,4}$. A ocorrência das quedas também dependerá das condições de adequação dos ambientes do domicílio, e dos ambientes externos, em relação às necessidades especiais dos idosos ${ }^{3}$.

As quedas podem provocar comprometimentos mais ou menos graves na vida dos idosos, e não raro podem levar a óbito. Porém, mesmo a ocorrência de quedas com lesões de menor gravidade, sem risco de provocar óbito ou deixar sequelas, pode produzir impacto significativo na qualidade de vida do idoso ${ }^{5,6}$ e levar ao desenvolvimento do medo de cair ${ }^{7}$.

O impacto das quedas na qualidade de vida pode ser abordada em uma perspectiva mais geral, em que são considerados fatores relacionados à condição de vida, como renda, trabalho e moradia, ou na perspectiva da qualidade de vida relacionada à saúde (QVRS), em que são avaliados os impactos que as doenças ou agravos de saúde causam na vida das pessoas ${ }^{8,9}$. A mensuração da qualidade de vida em saúde pode ser feita por meio de instrumentos estruturados, e um dos mais utilizados é o The Medical Outcomes Study 36- Item Short-Form Health Survey (SF-36), que gera informações sobre oito domínios de saúde: capacidade funcional, aspectos físicos, dor, estado geral de saúde, vitalidade, aspectos sociais e emocionais e saúde mental ${ }^{10,11}$.

Muitas pesquisas sobre quedas em idosos foram publicadas no Brasil e em outros países, mas poucas avaliaram, em base populacional, o impacto delas na QVRS de idosos, seja no Brasil ${ }^{12,13}$ ou em outros países ${ }^{5,6,14,15}$. Esses estudos detectaram prejuízos provocados pelas quedas, em especial nos domínios de aspectos físicos, capacidade funcional e saúde geral.

Entretanto, é preciso reconhecer que as quedas constituem um conjunto de acidentes bastante heterogêneo quanto às suas características. Elas se diferenciam com relação à frequência anual dos acidentes, ao local de ocorrência, às circunstâncias que as provocaram e ao grau de limitações que acarretam ${ }^{7,16}$. Estudos revelam que de $11 \%^{17}$ a $41,1 \%^{16}$ dos idosos haviam sofrido mais de uma queda num período de 12 meses. Quanto ao local de ocorrência, os estudos apontam que são mais frequentes no ambiente doméstico ${ }^{7,16,18}$. E em relação às circunstâncias, estudo realizado em município brasileiro apontou que $28,9 \%$ das quedas dos idosos haviam resultado de tontura ou vertigem ${ }^{7}$. O percentual de presença de limitações por elas provocadas nas atividades de vida diária variou entre os estudos: $13,6 \%^{7}$ e $45,1 \%^{16}$.

Entre as poucas pesquisas que avaliaram o impacto das quedas na QVRS de idosos, apenas quatro consideraram a influência de alguma característica da queda no grau do comprometimento provocado, sendo que três desses estudos analisaram o impacto segundo o número de quedas (ou quedas recorrentes) ${ }^{5,12,14}$, e o quarto estudo avaliou os declínios dos escores de acordo com o grau de limitação relatado ${ }^{13}$. Desses, apenas dois são brasileiros ${ }^{12,13}$.

Entende-se que, no contexto atual de acelerado envelhecimento populacional, a ocorrência de quedas de idosos tende a ser crescente e com consequências relevantes para a saúde e a qualidade de vida, tendo impacto significativo nos gastos impostos ao sistema de saúde ${ }^{19}$. Nesse cenário, constatando a escassez de estudos de base populacional que se propuseram a avaliar a influência das quedas na qualidade de vida segundo as características do evento, este estudo teve por objetivo avaliar a QVRS segundo a frequência e as características das quedas em idosos.

\section{Métodos}

Este é um estudo transversal, de base populacional, realizado com idosos não institucionalizados, residentes na área urbana do município de Campinas, São Paulo. Os dados são provenientes de inquérito domiciliar de saúde (ISACamp) realizado em 2014/ 2015.

Para a seleção dos sujeitos do inquérito, foi utilizada amostragem probabilística, estratificada, 
por conglomerados e realizada em dois estágios. No primeiro foram sorteados 70 setores censitários, e no segundo foram selecionados domicílios por meio de sorteio sistemático aplicado às relações atualizadas dos domicílios existentes nos 70 setores sorteados. O tamanho da amostra foi definido de forma a possibilitar a estimativa de uma proporção de 0,50 , com intervalo de confiança de $95 \%(z=1,96)$, com erro de amostragem de $4 \%$ e considerando um efeito de delineamento igual a dois. Assim, foi definida uma amostra de 1.000 idosos e, para alcançar esse tamanho amostral, foram selecionados, de forma independente, 3.157 domicílios, já considerando os percentuais de perdas esperados ${ }^{20}$. Outras informações sobre o processo amostral podem ser obtidas por meio do endereço eletrônico: https://fcm.unicamp.br/ $\mathrm{fcm} /$ sites/default/files/2018/page/plano_de_amostragem_isacamp_2014.15.pdf.

Os dados do ISACamp foram obtidos por meio de questionário estruturado, organizado em 12 blocos temáticos, e aplicado em entrevistas domiciliares que foram realizadas com o uso de tablets por entrevistadores treinados e supervisionados.

Para este estudo, foram analisadas, como variáveis dependentes, as oito escalas/domínios do SF-36 (versão 2) (capacidade funcional, aspectos físicos, dor, estado geral de saúde, vitalidade, aspectos emocionais, aspectos sociais e saúde mental) e os dois componentes (físico e mental) que resumem as escalas. A versão 2 do SF-36 foi validada no Brasil por Laguardia ${ }^{21}$.

Para obter os escores do SF-36, de acordo com a metodologia proposta para o instrumento ${ }^{10}$, foram atribuídas pontuações a cada questão, conforme a resposta do entrevistado. Os escores obtidos em cada domínio foram convertidos para uma escala de zero a 100, sendo que zero corresponde ao pior estado de saúde, e 100 ao melhor ${ }^{10}$.

As variáveis independentes analisadas foram:

Ocorrência de quedas, obtida por meio da pergunta: "O(a) sr.(a) sofreu alguma queda, seja leve ou grave, nos últimos 12 meses?”, com respostas sim ou não.

Número de quedas sofridas nos últimos 12 meses, categorizado em: uma ou duas e três ou mais.

Em relação à queda mais grave sofrida nos últimos 12 meses, foram analisadas as variáveis:

Causa da queda: categorizada em desmaio/ tontura e escorregou/tropeçou.

Local da queda: categorizado em domicílio, rua e outros locais (escola, clube, praça de esporte, academia ou outro).
Relato de limitação provocada pelas quedas ( $\operatorname{sim} /$ não), obtida por meio da pergunta: "Essa queda limitou as suas atividades habituais?"

As variáveis utilizadas para os ajustes das análises foram sexo, faixa etária e número de doenças crônicas, considerando aquelas presentes no checklist e referidas como diagnosticadas por médico ou outro profissional de saúde: hipertensão arterial, diabetes, angina, infarto do miocárdio, tumor/câncer, artrite/reumatismo/artrose, osteoporose, asma/bronquite/enfisema, rinite, sinusite, tendinite/lesão por esforço repetitivo, varizes de membros inferiores, acidente vascular cerebral, colesterol elevado, doença de coluna/ problema de coluna.

Foram estimadas médias, erro padrão e intervalos de confiança de 95\% dos escores de cada uma das oito escalas e para cada componente do SF-36. A associação entre QVRS e quedas foi verificada com o uso de regressão linear simples e múltipla, estimando os betacoeficientes, com ajustes pelas variáveis sexo, faixa etária e número de morbidades. Todas as análises foram realizadas por meio do software Stata 15.0 (StataCorp, College Station, Estados Unidos), utilizando os comandos svy, que permitem incorporar as ponderações necessárias em decorrência do desenho amostral complexo.

O projeto do ISACamp 2014/2015 foi aprovado pelo Comitê de Ética em Pesquisa (CEP) da Universidade Estadual de Campinas. O presente estudo também foi aprovado pelo CEP, via Plataforma Brasil.

\section{Resultados}

Entre os domicílios que haviam sido sorteados para a amostra de idosos, as perdas totalizaram $6,8 \%$, considerando-se as recusas e não encontrar o morador após mais de três visitas. Dos 1.168 idosos encontrados nos domicílios sorteados, houve $14 \%$ de recusas em participar da pesquisa e $1,5 \%$ de perdas por outros motivos, de forma que a amostra estudada ficou constituída por 986 idosos.

A prevalência de quedas foi de $17,1 \%$ (IC: $14,76-19,81$ ), sendo que $67,5 \%$ dos idosos haviam sofrido uma única queda, $15,3 \%$ duas e $17,1 \%$ três ou mais quedas nos 12 meses anteriores à entrevista. Entre os tipos de quedas, 8,2\% foram decorrentes de desmaio ou tontura e 91,7\% de escorregão ou tropeção. Quanto ao local da principal queda sofrida, constatou-se que 53,3\% delas haviam ocorrido no domicílio $(20,6 \%$ no 
quintal, $8,5 \%$ no quarto, $6,8 \%$ na cozinha, $5,6 \%$ no banheiro), $35,5 \%$ em via pública e $11,1 \%$ em outros locais. Com relação às limitações, 32,4\% relataram ter tido as atividades diárias prejudicadas pela queda.

As análises ajustadas por sexo, idade e número de morbidades revelaram que os idosos que sofreram quedas no ano anterior à pesquisa apresentaram redução das médias dos escores de QVRS nos domínios de capacidade funcional, aspectos físicos e dor (Tabela 1).

Os idosos que relataram uma ou duas quedas tiveram declínio da média do escore apenas na escala de dor, enquanto aqueles que sofreram três ou mais quedas tiveram redução, e de grande magnitude, em seis domínios do SF-36. O com maior declínio foi o domínio de aspectos físicos, seguido pelo de aspectos emocionais (Tabela 2).

Os prejuízos provocados pelas quedas foram de maior magnitude nos idosos que relataram quedas por desmaio ou tontura. Eles apresentaram declínio nas médias de QVRS nos domínios de capacidade funcional, aspectos físicos e aspec- tos emocionais, e aqueles que sofreram quedas devido a escorregões ou tropeços tiveram redução de escore nos domínios de capacidade funcional e dor. Os tamanhos dos declínios, exceto para dor, foram mais elevados entre os idosos que relataram quedas por desmaio ou tontura (Tabela 3).

Os idosos que sofreram quedas no domicílio apresentaram prejuízos em quatro domínios do SF-36, sendo que nas escalas de capacidade funcional e aspectos físicos foram observados os maiores declínios. Já os idosos que sofreram quedas na rua ou em outros locais externos ao domicílio apresentaram redução de escore apenas no domínio de dor (Tabela 4).

Nas quedas sem relato de limitações das atividades habituais, constatou-se redução significativa apenas no escore do domínio de dor, enquanto nos idosos que relataram limitações provocadas pelas quedas foram detectados prejuízos em quatro domínios (capacidade funcional, aspectos físicos, dor, aspectos emocionais) do SF-36 (Tabela $5)$.
Tabela 1. Médias e beta coeficientes dos escores dos domínios do SF-36 segundo a ocorrência de quedas. Campinas, São Paulo, Brasil.

\begin{tabular}{|c|c|c|c|c|}
\hline \multirow[b]{2}{*}{$\begin{array}{c}\text { Domínios e } \\
\text { zcomponentes } \\
\text { do SF-36 }\end{array}$} & \multicolumn{2}{|c|}{$\begin{array}{c}\text { Médias } \\
\text { (erro padrão) }\end{array}$} & \multirow[b]{2}{*}{$\begin{array}{c}\boldsymbol{\beta} \\
(\text { valor } \\
\text { de p) }\end{array}$} & \multirow[b]{2}{*}{$\begin{array}{c}\beta^{\star} \\
\text { (valor } \\
\operatorname{dep} \text { ) }\end{array}$} \\
\hline & $\begin{array}{c}\text { Não } \\
\text { sofreu } \\
\text { quedas } \\
(\mathbf{n}=\mathbf{8 1 0})\end{array}$ & $\begin{array}{c}\text { Sofreu } \\
\text { quedas } \\
(n=174)\end{array}$ & & \\
\hline $\begin{array}{l}\text { Capacidade } \\
\text { funcional }\end{array}$ & $\begin{array}{l}72,1 \\
(1,5)\end{array}$ & $\begin{array}{l}60,0 \\
(2,8)\end{array}$ & $\begin{array}{c}-12,1 \\
(0,000)\end{array}$ & $\begin{array}{c}-6,1 \\
(0,007)\end{array}$ \\
\hline $\begin{array}{l}\text { Aspectos } \\
\text { físicos }\end{array}$ & $\begin{array}{l}76,2 \\
(1,5)\end{array}$ & $\begin{array}{l}66,0 \\
(3,1)\end{array}$ & $\begin{array}{c}-10,2 \\
(0,002)\end{array}$ & $\begin{array}{c}-5,8 \\
(0,046)\end{array}$ \\
\hline Dor & $\begin{array}{l}73,4 \\
(1,3)\end{array}$ & $\begin{array}{l}61,3 \\
(2,7)\end{array}$ & $\begin{array}{c}-12,1 \\
(0,000)\end{array}$ & $\begin{array}{c}-7,6 \\
(0,003)\end{array}$ \\
\hline Saúde geral & $\begin{array}{l}73,5 \\
(1,1)\end{array}$ & $\begin{array}{l}69,7 \\
(1,8)\end{array}$ & $\begin{array}{c}-3,8 \\
(0,036)\end{array}$ & $\begin{array}{c}-0,4 \\
(0,802)\end{array}$ \\
\hline Vitalidade & $\begin{array}{l}73,0 \\
(1,2)\end{array}$ & $\begin{array}{l}67,8 \\
(2,3)\end{array}$ & $\begin{array}{c}-5,2 \\
(0,028)\end{array}$ & $\begin{array}{c}-1,3 \\
(0,560)\end{array}$ \\
\hline $\begin{array}{l}\text { Aspectos } \\
\text { emocionais }\end{array}$ & $\begin{array}{l}83,8 \\
(1,3)\end{array}$ & $\begin{array}{l}76,6 \\
(2,8)\end{array}$ & $\begin{array}{c}-7,2 \\
(0,012)\end{array}$ & $\begin{array}{c}-4,0 \\
(0,130)\end{array}$ \\
\hline $\begin{array}{l}\text { Aspectos } \\
\text { sociais }\end{array}$ & $\begin{array}{l}84,4 \\
(1,0)\end{array}$ & $\begin{array}{l}80,7 \\
(2,4)\end{array}$ & $\begin{array}{c}-3,7 \\
(0,131)\end{array}$ & $\begin{array}{c}-0,1 \\
(0,950)\end{array}$ \\
\hline Saúde mental & $\begin{array}{l}76,9 \\
(0,9)\end{array}$ & $\begin{array}{l}71,7 \\
(1,8)\end{array}$ & $\begin{array}{c}-5,2 \\
(0,005)\end{array}$ & $\begin{array}{c}-2,3 \\
(0,235)\end{array}$ \\
\hline
\end{tabular}

* Análise ajustada por sexo, idade e número de morbidades.

Fonte: ISACamp 2014/2015.
Tabela 2. Médias e beta coeficientes ${ }^{\mathrm{a}}$ dos escores dos domínios do SF-36 segundo número de quedas. Campinas, São Paulo, Brasil.

\begin{tabular}{|c|c|c|c|c|}
\hline \multirow[b]{2}{*}{$\begin{array}{l}\text { Domínios e } \\
\text { componentes } \\
\text { do SF-36 }\end{array}$} & \multicolumn{2}{|c|}{$\beta$ (valor de p) } & \multicolumn{2}{|c|}{$\boldsymbol{\beta}^{*}($ valor de p) } \\
\hline & $\begin{array}{c}\text { Uma } \\
\text { ou duas } \\
\text { quedas } \\
(\mathrm{n}=145)\end{array}$ & $\begin{array}{c}\text { Três } \\
\text { quedas } \\
\text { ou mais } \\
(\mathbf{n}=29)\end{array}$ & $\begin{array}{c}\text { Uma } \\
\text { queda } \\
\text { ou duas } \\
\text { quedas }^{*}\end{array}$ & $\begin{array}{c}\text { Três } \\
\text { quedas } \\
\text { ou } \\
\text { mais }\end{array}$ \\
\hline $\begin{array}{l}\text { Capacidade } \\
\text { funcional }\end{array}$ & $\begin{array}{c}-9,2 \\
(0,002)\end{array}$ & $\begin{array}{c}-26,3 \\
(0,000)\end{array}$ & $\begin{array}{c}-4,3 \\
(0,059)\end{array}$ & $\begin{array}{c}-14,7 \\
(0,002)\end{array}$ \\
\hline $\begin{array}{l}\text { Aspectos } \\
\text { físicos }\end{array}$ & $\begin{array}{c}-6,1 \\
(0,049)\end{array}$ & $\begin{array}{c}-30,2 \\
(0,000)\end{array}$ & $\begin{array}{c}-2,4 \\
(0,368)\end{array}$ & $\begin{array}{c}-22,2 \\
(0,001)\end{array}$ \\
\hline Dor & $\begin{array}{c}-10,6 \\
(0,000)\end{array}$ & $\begin{array}{c}-19,1 \\
(0,001)\end{array}$ & $\begin{array}{c}-6,7 \\
(0,010)\end{array}$ & $\begin{array}{c}-12,3 \\
(0,027)\end{array}$ \\
\hline Saúde & $\begin{array}{c}-3,3 \\
(0,086)\end{array}$ & $\begin{array}{c}-6,1 \\
(0,083)\end{array}$ & $\begin{array}{c}-0,3 \\
(0,853)\end{array}$ & $\begin{array}{c}-1,0 \\
(0,800)\end{array}$ \\
\hline Vital & $\begin{array}{c}-3,0 \\
(0,208)\end{array}$ & $\begin{array}{c}-15,4 \\
(0,001)\end{array}$ & $\begin{array}{c}0,4 \\
(0,853)\end{array}$ & $\begin{array}{c}-9,3 \\
(0,087)\end{array}$ \\
\hline $\begin{array}{l}\text { Aspectos } \\
\text { emocionais }\end{array}$ & $\begin{array}{c}-4,2 \\
(0,136)\end{array}$ & $\begin{array}{c}-21,9 \\
(0,002)\end{array}$ & $\begin{array}{c}-1,2 \\
(0,649)\end{array}$ & $\begin{array}{c}-17,7 \\
(0,022)\end{array}$ \\
\hline $\begin{array}{l}\text { Aspectos } \\
\text { sociais }\end{array}$ & $\begin{array}{c}-0,5 \\
(0,829)\end{array}$ & $\begin{array}{c}-19,2 \\
(0,002)\end{array}$ & $\begin{array}{c}3,1 \\
(0,137)\end{array}$ & $\begin{array}{c}-15,7 \\
(0,022)\end{array}$ \\
\hline Saúde mental & $\begin{array}{c}-2,8 \\
(0,105)\end{array}$ & $\begin{array}{c}-16,4 \\
(0,002)\end{array}$ & $\begin{array}{c}-0,1 \\
(0,948)\end{array}$ & $\begin{array}{c}-12,7 \\
(0,026)\end{array}$ \\
\hline
\end{tabular}
por sexo, idade e número de morbidades.

Fonte: ISACamp 2014/2015. 


\section{Discussão}

O estudo permitiu verificar que o impacto das quedas na qualidade de vida relacionada à saúde da população idosa depende da característica do evento. Os idosos com relato de três ou mais quedas no ano anterior, aqueles que caíram em decorrência de desmaio ou tontura, os que sofreram quedas no domicílio e os que relataram limitações provocadas pelos tombos foram os que apresentaram maiores declínios dos escores de QVRS.

Foram evidenciados prejuízos nas escalas de capacidade funcional, nos aspectos físicos e na dor no subgrupo da população idosa que sofreu quedas. Estudos realizados no Brasil ${ }^{13,22} \mathrm{e}$ em outros países ${ }^{5,6,14}$ também têm constatado, por meio de instrumentos estruturados, entre eles o SF-36, o European Quality of Life Group Instrument (EQ-5D) e o World Health Organization Quality of Life Instrument (WHOQOL), pior qualidade de vida em decorrência desses eventos.

Menores médias na escala de capacidade funcional indicam maior dificuldade para executar tarefas diárias simples, como deslocar-se, tomar banho ou vestir-se, enquanto o impacto no aspecto físico avalia as limitações provocadas pelos problemas de saúde nas atividades de trabalho ou em outras atividades que o idoso desempenhe ${ }^{10}$. Essas dificuldades podem decorrer de lesões, escoriações e fraturas provocadas pelas quedas, e podem levar à redução da mobilidade ${ }^{23}$. Além disso, as tarefas diárias podem estar sendo prejudicadas pelo declínio da autoconfiança, pelo medo de novas quedas ou por atitudes protetoras de familiares e cuidadores ${ }^{15,24}$. A associação com o domínio de dor, que avalia a presença dela nas últimas quatro semanas e o quanto interferiu no trabalho realizado dentro ou fora de casa ${ }^{10}$, decorre das lesões provocadas pelas quedas, como as mencionadas anteriormente, além de hematomas e contusões ${ }^{23}$.

Os idosos que sofreram três ou mais quedas apresentaram comprometimento em seis domínios do SF-36, sendo os aspectos físicos, emocionais e sociais e a capacidade funcional os domínios mais fortemente prejudicados, enquanto aqueles que relataram uma ou duas quedas tiveram prejuízo apenas no domínio de dor. Estudo que acompanhou uma coorte de idosos por sete anos na Alemanha detectou que as quedas recorrentes provocaram declínio mais substancial à QV, avaliada por meio do EQ-5D, em comparação às quedas únicas ${ }^{5}$. Em pesquisa realizada em Taiwan também ficou constatado que idosos que
Tabela 3. Médias e beta coeficientes ${ }^{\mathrm{a}}$ dos escores dos domínios do SF-36 segundo tipo de queda. Campinas, São Paulo, Brasil.

\begin{tabular}{lcccc}
\hline \multirow{2}{*}{$\begin{array}{c}\text { Domínios e } \\
\text { componentes }\end{array}$ do SF-36 } & $\begin{array}{c}\boldsymbol{\beta} \text { (valor de p) } \\
\text { Escorregou/ } \\
\text { tropeçou } \\
(\mathbf{n}=\mathbf{1 5 1})\end{array}$ & $\begin{array}{c}\text { Desmaio/ } \\
\text { tontura } \\
(\mathbf{n = 1 7 )}\end{array}$ & $\begin{array}{c}\text { Escorregou/ } \\
\text { tropeçou }\end{array}$ & $\begin{array}{c}\text { Desmaio/ } \\
\text { tontura }\end{array}$ \\
\hline Capacidade & $-10,3$ & $-27,3$ & $-5,1(0,031)$ & $-15,8$ \\
funcional & $(0,000)$ & $(0,005)$ & & $(0,029)$ \\
Aspectos & $-7,5(0,018)$ & $-33,4$ & $-3,7(0,211)$ & $-26,2$ \\
Físicos & & $0,001)$ & & $(0,06)$ \\
Dor & $-12,4$ & $-7,3$ & $-8,6(0,002)$ & $-3,5$ \\
& $(0,000)$ & $(0,280)$ & & $(0,649)$ \\
Saúde geral & $-3,0(0,119)$ & $-13,6$ & $0,2(0,934)$ & $-8,9$ \\
& & $(0,004)$ & & $(0,057)$ \\
Vitalidade & $-3,7(0,130)$ & $-17,1$ & $-0,1(0,977)$ & $-11,7$ \\
& & $(0,011)$ & & $(0,099)$ \\
Aspectos & $-5,1(0,066)$ & $-22,8$ & $-2,1(0,425)$ & $-20,6$ \\
Emocionais & & $(0,009)$ & & $(0,026)$ \\
Aspectos & $-1,8(0,461)$ & $-19,1$ & $1,1(0,616)$ & $-10,1$ \\
Sociais & & $(0,006)$ & & $(0,189)$ \\
Saúde mental & $-4,3(0,016)$ & $-10,8$ & $-1,5(0,438)$ & $-6,8$ \\
& & $(0,039)$ & & $(0,216)$ \\
\hline
\end{tabular}

${ }^{a}$ Categoria de comparação: não sofreu quedas; ${ }^{\star}$ análise ajustada por sexo, idade e número de morbidades.

Fonte: ISACamp 2014/2015.

Tabela 4. Médias e beta coeficientes ${ }^{\mathrm{a}}$ dos escores dos domínios do SF-36 segundo local de ocorrência das quedas. Campinas, São Paulo, Brasil.

\begin{tabular}{lcccc}
\hline \multirow{2}{*}{$\begin{array}{c}\text { Domínios e } \\
\text { componentes }\end{array}$} & \multicolumn{2}{c}{$\boldsymbol{\beta}$ (valor de $\mathbf{p})$} & \multicolumn{2}{c}{$\boldsymbol{\beta}^{\star}$ (valor de p) } \\
\cline { 2 - 5 } & $\begin{array}{c}\text { Na rua } \\
\text { e outros } \\
(\mathbf{n}=\mathbf{7 2})\end{array}$ & $\begin{array}{c}\text { No } \\
\text { domicílio } \\
(\mathbf{n}=\mathbf{1 0 2})\end{array}$ & $\begin{array}{c}\text { Na } \\
\text { rua e } \\
\text { outros }\end{array}$ & $\begin{array}{c}\text { No } \\
\text { domicílio }\end{array}$ \\
\hline Capacidade & 0,7 & $-23,8$ & 2,3 & $-13,7$ \\
funcional & $(0,831)$ & $(0,000)$ & $(0,450)$ & $(0,000)$ \\
Aspectos & 0,8 & $-19,8$ & 1,4 & $-12,2$ \\
físicos & $(0,835)$ & $(0,000)$ & $(0,658)$ & $(0,004)$ \\
Dor & $-9,8$ & $-14,2$ & $-7,2$ & $-9,6$ \\
& $(0,000)$ & $(0,000)$ & $(0,043)$ & $(0,003)$ \\
Saúde geral & $-2,3$ & $-5,2$ & $-0,3$ & $-0,6$ \\
& $(0,318)$ & $(0,023)$ & $(0,908)$ & $(0,789)$ \\
Vitalidade & $-1,8$ & $-8,3$ & $-0,03$ & $-2,5$ \\
& $(0,568)$ & $(0,004)$ & $(0,991)$ & $(0,389)$ \\
Aspectos & 1,0 & $-14,5$ & 2,4 & $-9,8$ \\
emocionais & $(0,782)$ & $(0,000)$ & $(0,505)$ & $(0,009)$ \\
Aspectos & $-0,5$ & $-6,6$ & 1,2 & $-1,3$ \\
sociais & $(0,878)$ & $(0,031)$ & $(0,730)$ & $(0,639)$ \\
Saúde mental & $-5,0$ & $-5,4$ & $-2,8$ & $-1,9$ \\
& $(0,073)$ & $(0,015)$ & $(0,325)$ & $(0,426)$ \\
\hline
\end{tabular}

${ }^{a}$ Categoria de comparação: não sofreu quedas; * análise ajustada por sexo, idade e número de morbidades.

Fonte: ISACamp 2014/2015. 
Tabela 5. Médias e beta coeficientes ${ }^{\mathrm{a}}$ dos escores dos domínios do SF-36 segundo limitação provocada pelas quedas. Campinas, São Paulo, Brasil.

\begin{tabular}{|c|c|c|c|c|}
\hline \multirow[b]{2}{*}{$\begin{array}{c}\text { Domínios e } \\
\text { componentes } \\
\text { do SF-36 }\end{array}$} & \multicolumn{2}{|c|}{$\beta$ (valor de p) } & \multicolumn{2}{|c|}{$\beta^{\star}($ valor de $p)$} \\
\hline & $\begin{array}{c}\text { Sofreu } \\
\text { quedas } \\
\text { e não } \\
\text { limitou } \\
(\mathrm{n}=113)\end{array}$ & $\begin{array}{c}\begin{array}{c}\text { Sofreu } \\
\text { quedas }\end{array} \\
\mathrm{e} \\
\text { limitou } \\
(\mathrm{n}=61)\end{array}$ & $\begin{array}{c}\text { Sofreu } \\
\text { quedas } \\
\text { e não } \\
\text { limitou* }^{*}\end{array}$ & $\begin{array}{c}\text { Sofreu } \\
\text { quedas e } \\
\text { limitou }^{\star}\end{array}$ \\
\hline $\begin{array}{l}\text { Capacidade } \\
\text { funcional }\end{array}$ & $\begin{array}{c}-5,9 \\
(0,067)\end{array}$ & $\begin{array}{c}-24,7 \\
(0,000)\end{array}$ & $\begin{array}{c}-1,9 \\
(0,506)\end{array}$ & $\begin{array}{c}-14,4 \\
(0,000)\end{array}$ \\
\hline $\begin{array}{l}\text { Aspectos } \\
\text { físicos }\end{array}$ & $\begin{array}{c}-4,2 \\
(0,210)\end{array}$ & $\begin{array}{c}-22,8 \\
(0,000)\end{array}$ & $\begin{array}{c}-1,4 \\
(0,691)\end{array}$ & $\begin{array}{c}-15,1 \\
(0,003)\end{array}$ \\
\hline Dor & $\begin{array}{c}-8,4 \\
(0,007)\end{array}$ & $\begin{array}{c}-19,9 \\
(0,000)\end{array}$ & $\begin{array}{c}-5,6 \\
(0,046)\end{array}$ & $\begin{array}{c}-14,4 \\
(0,002)\end{array}$ \\
\hline Saúde geral & $\begin{array}{c}-1,5 \\
(0,005)\end{array}$ & $\begin{array}{c}-8,7 \\
(0,005)\end{array}$ & $\begin{array}{c}1,3 \\
(0,559)\end{array}$ & $\begin{array}{c}-4,3 \\
(0,138)\end{array}$ \\
\hline Vitalidade & $\begin{array}{c}-1,8 \\
(0,455)\end{array}$ & $\begin{array}{c}-12,4 \\
(0,001)\end{array}$ & $\begin{array}{c}0,7 \\
(0,750)\end{array}$ & $\begin{array}{c}-5,7 \\
(0,120)\end{array}$ \\
\hline $\begin{array}{l}\text { Aspectos } \\
\text { emocionais }\end{array}$ & $\begin{array}{c}-0,6 \\
(0,820)\end{array}$ & $\begin{array}{c}-21,0 \\
(0,000)\end{array}$ & $\begin{array}{c}1,0 \\
(0,731)\end{array}$ & $\begin{array}{c}-14,7 \\
(0,006)\end{array}$ \\
\hline $\begin{array}{l}\text { Aspectos } \\
\text { sociais }\end{array}$ & $\begin{array}{c}-0,7 \\
(0,793)\end{array}$ & $\begin{array}{c}-10,0 \\
(0,011)\end{array}$ & $\begin{array}{c}1,7 \\
(0,526)\end{array}$ & $\begin{array}{c}-4,1 \\
(0,299)\end{array}$ \\
\hline Saúde mental & $\begin{array}{c}-4,3 \\
(0,038)\end{array}$ & $\begin{array}{c}-7,3 \\
(0,022)\end{array}$ & $\begin{array}{c}-2,1 \\
(0,318)\end{array}$ & $\begin{array}{c}-2,7 \\
(0,417)\end{array}$ \\
\hline
\end{tabular}

${ }^{a}$ Categoria de comparação: não sofreu quedas; ${ }^{*}$ análise ajustada por sexo, idade e número de morbidades.

Fonte: ISACamp 2014/2015.

sofreram tombos recorrentes apresentaram menores médias de QV em todas as escalas do SF-36, em comparação àqueles que caíram apenas uma vez ${ }^{14}$. As quedas recorrentes, em decorrência das lesões provocadas, levariam os idosos à maior limitação na realização de tarefas usuais e nas atividades diárias, itens avaliados nos domínios de capacidade funcional e aspectos físicos, respectivamente ${ }^{10}$. Embora as análises tenham sido ajustadas por idade, sexo e número de morbidades, idosos mais debilitados, e assim com pior QVRS, estariam também mais propensos a sofrer quedas recorrentes, aspecto que deve ser considerado neste estudo, que é de corte transversal.

Chama a atenção, em relação às quedas recorrentes, os significativos prejuízos detectados nos aspectos emocionais e sociais e na saúde mental. Estudo realizado com idosos de Taiwan ${ }^{14}$ também revelou que o impacto das quedas na QVRS vai além daquele relacionado ao físico, gerando também prejuízos nas escalas de aspectos emocionais, sociais e mentais. Dessa forma, alerta- se para a necessidade de considerar os aspectos emocionais nas ações de cuidado aos idosos que sofrem quedas recorrentes.

Quanto à circunstância das quedas, os estudos revelam maior frequência das provocadas por perda de equilíbrio resultantes de tropeços e escorregões ${ }^{7,16}$. Entretanto, as pesquisas não têm investigado o efeito das circunstâncias das quedas nos prejuízos sobre a QV. Os achados da presente pesquisa, que revela que os idosos que caíram devido a desmaio ou tontura apresentaram declínios intensos nos escores de capacidade funcional, aspectos físicos e também emocionais, com redução muito maiores do que os que sofreram quedas por tropeçarem ou escorregarem, apontam a necessidade de atentar para a circunstância da queda. A tontura ou o desmaio, provenientes do mau funcionamento dos mecanismos de controle postural decorrentes do processo de envelhecimento, somados às doenças crônicodegenerativas, têm sido associados a quedas na população idosa ${ }^{18,25,26,27}$. A queixa de tontura/ desmaio se relaciona à sensação de flutuação, vertigem, desorientação corporal, provenientes de alterações sensoriais entre os sistemas visuais, vestibular e proprioceptivos, que influenciam na regulação do funcionamento dos mecanismos de controle da estabilidade e orientação postural ${ }^{27,28}$. A queda com preservação da consciência possibilita utilizar mecanismos de defesa e de proteção contra lesões, ausentes nos tombos por desmaio. Saber se a queda ocorreu por tontura ou desmaio é fundamental para buscar diagnosticar, e se possível tratar, as causas. Embora as análises tenham sido ajustadas pela idade e pelo número de doenças crônicas, é preciso considerar que os indivíduos que sofreram quedas devido a tontura ou desmaio podem apresentar outras vulnerabilidades e condições de saúde desfavoráveis, não levadas em conta nos ajustes. Já as quedas por escorregão/tropeços são comuns entre os idosos e a associação com o domínio de dor se relaciona com as consequências diretas geradas pelo evento, tais como lesões e contusões ${ }^{29}$. Tombos por escorregão ou tropeção são alertas para a avaliação das condições do ambiente de convívio do idoso e da necessidade de adaptações no local em que o evento ocorreu.

Quanto ao local de ocorrência das quedas, os dados da presente pesquisa evidenciaram que aquelas que ocorrem no domicílio tiveram maior impacto na QVRS do que as que aconteceram na rua ou em outros locais, em análises ajustadas por idade, sexo e número de doenças crônicas. Estudos sobre quedas em idosos têm constado 
que elas são mais frequentes no ambiente doméstico ${ }^{29,30}$, local em que as pessoas tendem cada vez mais, com o avançar da idade, a desenvolver suas atividades ${ }^{24}$. É possível que o local de ocorrência das quedas se associe à condição de saúde dos idosos, pois aqueles com idade avançada e mais fragilizados tendem a ficar mais em casa, enquanto idosos mais jovens e ativos se expõem mais a sofrerem quedas em ambientes abertos ${ }^{31}$ ou em outros locais fechados externos ao domicílio. Entretanto, não foram identificados estudos que tenham analisado a influência do local de ocorrência da queda no impacto na QV.

As quedas no ambiente doméstico são associadas às condições com que os idosos têm que lidar neste contexto, tais como piso escorregadio, tapetes, degraus nas soleiras da portas e ausência de apoios estrategicamente instalados ${ }^{30}$. A frequência mais elevada de quedas no ambiente doméstico e o maior impacto que elas ocasionam na qualidade de vida dos idosos, como detectado neste estudo, reforçam a necessidade de identificar as condições nas moradias que estejam inapropriadas para a segurança dos ido$\operatorname{sos}^{30}$. Orientações sobre esses aspectos, fornecidas pelos profissionais de saúde e divulgadas em programas educacionais, são fundamentais para destacar a enfatizar a necessidade de alterações/ adaptações nos domicílios e em outros locais, de forma a propiciar um ambiente mais seguro para os idosos ${ }^{32}$.

As médias dos escores das escalas de capacidade funcional, aspectos físicos, dor e aspectos emocionais declinaram significativamente nos idosos que relataram quedas com limitações posteriores. A limitação reportada pelo idoso de fato concordou com os achados obtidos pelo instrumento SF-36. As limitações se relacionam com a gravidade das quedas, e apenas um estudo de base populacional investigou o impacto do grau de limitações provocado por elas na QVRS, e constatou prejuízos nas escalas de capacidade funcional e de aspectos físicos, emocionais e sociais do SF$36^{13}$. As lesões provocadas pelas quedas são responsáveis pelo aumento da dependência para a realização das atividades da vida diária e, com o medo de novos episódios ${ }^{33,34}$, afetam também os aspectos emocionais dos idosos.

O domínio aspecto emocional apresentouse fortemente comprometido nos indivíduos que relataram três ou mais quedas $(\beta=-17,7)$, que sofreram quedas por desmaios/tontura $(\beta=$ $-20,6)$, com limitações das atividades $(\beta=-14,7)$ e nos domicílios $(\beta=-9,8)$. O comprometimento do domínio de saúde mental foi detectado apenas no segmento de idosos que sofreram três ou mais quedas. Pesquisa que investigou o impacto das quedas na QVRS também observou prejuízo no domínio emocional nos idosos que as sofreram ${ }^{6}$, e outro estudo encontrou essa associação apenas nos idosos com tombos recorrentes ${ }^{14}$. Dessa maneira, é importante considerar que as quedas são responsáveis por impactar não só a saúde física, mas os aspectos relacionados à saúde emocional. $\mathrm{O}$ conhecimento de tais consequências alerta para a necessidade de avaliar e considerar esses aspectos no acompanhamento dos idosos que sofreram quedas.

As análises dos resultados apresentados precisam levar em conta algumas limitações. Trata-se de um estudo transversal, que não permite verificar relações causais entre as variáveis estudadas. Também é preciso considerar a possibilidade de viés de informação, pois os dados foram obtidos por meio de entrevistas, e de viés de memória, pelo fato de a questão sobre as quedas ser referente às ocorridas nos últimos 12 meses. Ressalta-se, porém, que essa questão indaga a respeito dos tombos leves ou graves nos últimos 12 meses, buscando estimular a lembrança do evento pelo idoso. Por outro lado, neste estudo foi analisada uma amostra representativa da população de idosos do município, os dados foram coletados de forma padronizada e supervisionada, e utilizou-se o SF-36, um instrumento validado no Brasil e muito utilizado em todo o mundo.

Com este estudo, foi possível verificar que os impactos nos domínios de QVRS dependem das características das quedas, e poucas pesquisas haviam analisado alguns desses aspectos. Destacase que os idosos que caíram três ou mais vezes, que sofrem quedas no domicílio, que caem por tontura ou desmaio e que relatam limitações decorrentes apresentaram maior número de domínios prejudicados e danos de maior magnitude, e entre os domínios afetados está o dos aspectos emocionais. Quedas na rua ou outros locais, com até duas ocorrências no ano e sem relato de limitação, provocaram prejuízo significativo apenas no domínio de dor, e as consequências de tropeçar ou escorregar ocasionaram danos no domínio de dor e em capacidade funcional. O comprometimento importante nos domínios de aspectos sociais e de saúde mental foi detectado apenas no segmento de idosos que sofreram três ou mais quedas no ano. Mas vale mencionar que, com a escassez de pesquisas que avaliaram o impacto das características das quedas nas diferentes dimensões que envolvem a qualidade de vida relacionada à saúde, novos estudos sobre o tema 
são necessários para contemplar a diversidade de tipos de tombos e a consistência dos achados.

Os resultados deste estudo evidenciam a importância de que os gestores, em ações políticas destinadas a melhorar a qualidade de vida da população idosa, considerem a questão das quedas e que estes eventos envolvem características diversas podendo afetar a qualidade de vida de maneira distinta nas várias dimensões que a compõe, exigindo estratégias mais direcionadas a cada situação. O estudo também fornece informações que precisam ser consideradas pelos profissionais de saúde no acolhimento e atendimento dos pacientes, a fim de orientar idosos, cuidadores e familiares quanto às condutas adequadas para a prevenção de novos episódios e para a redução dos prejuízos que as quedas provocam, inclusive nas dimensões sociais e emocionais da qualidade de vida.

\section{Colaboradores}

Paiva MM contribuiu na análise e interpretação dos dados e na escrita e revisão crítica do manuscrito. Lima MG colaborou na interpretação dos dados e na revisão crítica do manuscrito. Barros MBA trabalhou na concepção e orientação do trabalho, na interpretação dos dados e na revisão crítica do manuscrito. 
ceiving home help services: study protocol for a randomised controlled trial. BMC Health Serv Res 2017; 17(1):559.

3. Barbosa KTF, Fernandes MGM, Oliveira FMRL, Santos KFO, Pereira MA. Queda em idosos: associação com morbidade e capacidade funcional. Rev Enferm UFPE Line 2013; 7(8):5068-5075.

4. Bekibele CO, Gureje O. Fall incidence in a population of elderly persons in Nigeria. Gerontology 2010; 56(3):278-283.

5. Thiem U, Klaaben-Mielke R, Trampisch U, Moschny A, Pientka L, Hinrichs T. Falls and EQ-5D rated quality of life in community-dwelling seniors with concurrent chronic diseases: A cross-sectional study. Health Qual Life Outcomes 2014; 12(1):1-7.

6. Peeters GM, Jones M, Byles J, Dobson AJ. Long-term consequences of noninjurious and injurious falls on well-being in older women. J Gerontol A Biol Sci Med Sci 2015; 70(12):1519-1525.

7. Nascimento JS, Paiva MM, Tavares DMS. Avaliação das características das quedas entre idosos comunitários. Rev Enferm Atenção Saúde 2017; 6(1):95-106.

8. Minayo MCS, Hartz ZMA, Buss PM. Qualidade de vida e saúde: um debate necessário. Cien Saude Colet 2000; 5(1):7-18.

9. Seidl EMF, Zannon CMLC. Qualidade de vida e saúde: aspectos conceituais e metodológicos. Cad Saude Publica 2004; 20(2):580-588.

10. Ware JE, Kosinski M, Bjorner JB, Turner-Bowker DM, Gandek B, Maruish ME. User's Manual for the SF-36 Health Survey. Lincoln: Quality Metric Incorporated; 2007.

11. Ciconelli RM, Ferraz MB, Santos W, Meinão I, Quaresma MR. Tradução para a língua portuguesa e validação do questionário genérico de avaliação de qualidade de vida SF-36 (Brasil SF-36). Rev Bras Reumatol 1999; 39:143-150.

12. Pimentel WRT, Pagotto V, Nakatani AYK, Pereira LV, Menezes RL. Quedas e qualidade de vida: associação com aspectos emocionais em idosos comunitários. Geriatr Gerontol Aging 2015; 9(2):42-48.

13. Rodrigues IG, Lima MG, Barros MBA. Falls and health-related quality of life (SF-36) in elderly people ISAcamp 2008. Health 2013; 5(12):49-57.

14. Chang NT, Chi LY, Yang NP, Chou P. The impact of falls and fear of falling on health-related quality of life in taiwanese elderly. J Community Health Nurs 2010; 27(2):84-95

15. Stenhagen M, Ekström H, Nordell E, Elmståhl S. Accidental falls, health-related quality of life and life satisfaction: a prospective study of the general elderly population. Arch Gerontol Geriatr 2014; 58(1):95-100.

16. Moraes SA, Soares WJS, Lustosa LP, Bilton TL, Ferrioli E, Perracini MR. Características das quedas em idosos que vivem na comunidade: estudo de base populacional. Rev Bras Geriatr Gerontol 2017; 20(5):693-704.

17. Perracini MR, Ramos LR. Fall-related factors in a cohort of elderly community residents. Rev Saude Publica 2002; 36(6):709-716.

18. Rodrigues IG, Fraga GP, Barros MBA. Quedas em idosos: fatores associados em estudo de base populacional. Rev Bras Epidemiol 2014; 17(9):705-718.

19. Abreu DROM, Novaes ES, Oliveira RR, Mathias TAF, Marcon SS. Internação e mortalidade por quedas em idosos no Brasil: análise de tendência. Cien Saude Colet 2018; 23(4):1131-1141.

20. Sousa NFS, Lima MG, Cesar CLG, Barros MBA. Envelhecimento ativo: prevalência e diferenças de gênero e idade em estudo de base populacional. Cad Saúde Publica 2018; 34(11):e00173317.

21. Laguardia J, Campos MR, Travassos CM, Najar AL, Anjos LA, Vasconcellos MM. Psychometric evaluation sample of Brazilian households: results of the survey Pesquisa Dimensões Sociais das Desigualdades (PDSD), Brazil, 2008. Health Qual Life Outcomes 2011; 9(1):61.

22. Stival MM, Lima LR, Funghetto SS, Silva AO, Pinho DLM, Karnikowski MGO. Fatores associados à qualidade de vida de idosos que frequentam uma unidade de saúde do Distrito Federal. Rev Bras Geriatr e Gerontol 2014; 17(2):395-405.

23. Abreu DROM, Azevedo RCS, Silva AMC, Reiners AAO, Abreu HCA. Fatores associados à recorrência de quedas em uma coorte de idosos. Cien Saude Colet 2016; 21(11):3439-3446.

24. Brito TA, Fernandes MH, Coqueiro RS, Souza de Jesus C. Quedas e capacidade funcional em idosos longevos residentes em comunidade. Texto contexto-enferm 2013; 22(1):43-51.

25. Soares WJS, Moraes SA, Ferriolli E, Perracini MR. Fatores associados a quedas e quedas recorrentes em idosos: estudo de base populacional. Rev Bras Geriatr e Gerontol 2014; 17(1):49-60.

26. Ciorba A, Bianchini C, Scanelli G, Pala M, Zurlo A, Aimoni C. The impact of dizziness on quality-of-life in the elderly. Eur Arch Oto-Rhino-Laryngology 2017; 274(3):1245-1250.

27. Moraes SA, Soares WJD, Ferriolli E, Perracini MR. Prevalence and correlates of dizziness in community-dwelling older people: a cross sectional population based study. BMC Geriatr 2013;13:4.

28. Lencastre MC, Lins CD. Tonturas. In: Freitas EV, Py L. Tratado de geriatria e gerontologia. Rio de Janeiro: Editora Guanabara Koogan; 2016. p. 1659-1696.

29. Antes DL, D'Orsi E, Benedetti TRB. Circunstâncias e consequências das quedas em idosos de Florianópolis. Epifloripa Idoso 2009. Rev Bras Epidemiol 2013; 16(2):469-481.

30. Ribeiro AP, Souza ER, Atie S, Souza AC, Schilithz AO. A influência das quedas na qualidade de vida de idosos. Cien Saude Colet 2008; 13(4):1265-1273. 
31. Kelsey JL, Berry SD, Procter-Gray E, Quach L, Nguyen US, Li W, Kiel DP, Lipsitz LA, Hannan MT. Indoor and outdoor falls in older adults are different: the maintenance of balance, independent living, intellect, and Zest in the Elderly of Boston Study. J Am Geriatr Soc 2010; 58(11):2135-2141.

32. Nascimento JS, Tavares DMS. Prevalência e fatores associados a quedas em idosos. Texto contexto-enferm 2016; 25(2):e0360015.

33. Lopes RA, Dias RC. O impacto das quedas na qualidade de vida dos idosos. ConScientiae Saúde 2010; 9(3):504-509.

34. Maia BC, Viana PS, Arantes PMM, Alencar MA. Consequências das quedas em idosos vivendo na comunidade. Rev Bras Geriatr e Gerontol 2011; 14(2):381-393.

Artigo apresentado em 03/02/2019

Aprovado em 03/11/2019

Versão final apresentada em 05/11/2019

Editores-chefes: Romeu Gomes, Antônio Augusto Moura da Silva 